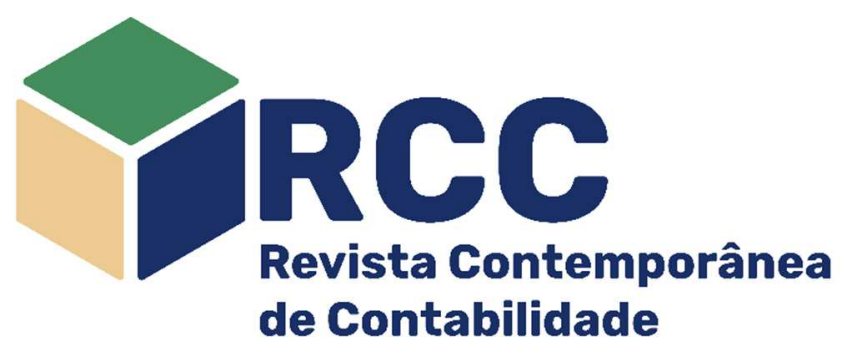

\title{
Diferenças de expectativas em auditoria no Brasil
}

\author{
Audit expectation gaps in Brazil
}

\section{Diferencias de expectativas en auditoría en Brasil}

\author{
Fernando Rocha Pereira \\ Doutorando em Controladoria e Contabilidade (UFMG) \\ Belo Horizonte/MG, Brasil \\ fernando.contabil@hotmail.com \\ https://orcid.org/0000-0002-6784-8677 (])
}

\begin{abstract}
Renata Turola Takamatsu*
Doutora em Controladoria e Contabilidade FEA (USP) Professora do Centro de Pós-Graduação e Pesquisas em Controladoria e Contabilidade do Departamento de Ciências Contábeis (UFMG), Belo Horizonte/MG, Brasil rettakamatsu@gmail.com https://orcid.org/0000-0002-0422-9895 ()

Laura Edith Taboada Pinheiro Doutora em Contabilidade e Finanças pela Universidad de Zaragoza Professora do Centro de Pós-Graduação e Pesquisas em Controladoria e Contabilidade do Departamento de Ciências Contábeis (UFMG), Belo Horizonte/MG, Brasil Itaboada@face.ufmg.b https://orcid.org/0000-0002-4511-7208
\end{abstract}

Endereço do contato principal para correspondência* Av. Antônio Carlos, 6627, CEP: 31270-901 - Belo Horizonte/MG, Brasil

\section{Resumo}

O trabalho examinou a situação das diferenças de expectativas de auditoria após a revisão das normas internacionais, que culminaram na aplicação do formato atualizado do Relatório dos Auditores no Brasil, no ano de 2017. Questionários aplicados resultaram em 185 respostas válidas para análises. O nível de exigência dos usuários da contabilidade em relação aos deveres dos auditores, captado pela proxy Índice de Expectativa (IE), foi calculado com base na metodologia desenvolvida por Porter (1993) e adaptado por Litjens, Buuren \& Vergoossen (2015), que abrange: i) gap de razoabilidade, (ii) gap de desempenho dos auditores e (iii) gap de padrões. O teste de Mann-Whitney revelou a perpetuação de evidências empíricas acerca das diferenças de expectativas documentadas nas últimas décadas. Assim, demonstrou-se que, mesmo analisando a percepção de auditores e usuários após a alteração da norma, que trouxe melhorias significativas ao relatório, diferenças de expectativas ainda podem ser detectadas. Testes adicionais indicaram que não foram encontradas diferenças significativas do índice de expectativas entre gêneros e diferentes níveis de utilização do relatório de auditoria.

Palavras-chave: Diferenças de expectativas em auditoria; Relatório dos auditores; Novo relatório dos auditores

\section{Abstract}

This paper examined the situation of audit expectations gaps after the review of international standards, which culminated in the application of the updated format of the Auditors' Report in Brazil, in 2017. Questionnaires applied resulted in 185 valid responses for analysis. The level of requirement of accounting users in relation to the duties of auditors, captured by the Expectation Index (EI) proxy, was calculated based on the methodology developed by Porter (1993) and adapted by Litjens, Buuren \& Vergoossen (2015), encompassing: i) reasonableness gap, (ii) auditor performance gap and (iii) standards gap. The Mann-Whitney test revealed the perpetuation of empirical evidence about the differences in expectations documented in recent decades. Thus, it was shown that, even analyzing the perception of auditors and users after changing the standard, which brought significant improvements to the report, expectation gaps can still be detected. Additional tests indicated that no significant differences were found in the expectations index between genders and different levels of use of the audit report.

Keywords: Audit expectation gaps; Auditors' report; New auditors report 


\section{Resumen}

El trabajo examinó la situación de las diferencias en las expectativas de auditoría después de la revisión de las normas internacionales que culminó con la aplicación del formato actualizado del Informe de los Auditores en Brasil en 2017. Los cuestionarios, aplicados en dos congresos nacionales y disponibles electrónicamente, dieron como resultado 185 respuestas válidas para analizar. El nivel de demanda de los usuarios de la contabilidad en relación con los deberes de los auditores, capturado por el Índice de Expectativas (IE), se calculó con base en la metodología desarrollada por Porter (1993), que cubre: i) brecha de razonabilidad, (ii) brecha de desempeño de los auditores y (iii) brecha de estándares. Mann-Whitney reveló la perpetuación de la evidencia empírica sobre las diferencias en expectativas documentadas en las últimas décadas. Así, se demostró que aun analizando la percepción de auditores y usuarios tras el cambio de norma, que trajo mejoras significativas al informe, aún se pueden detectar diferencias en las expectativas. Pruebas adicionales indicaron que no hubo diferencias significativas en el índice de expectativas entre los géneros y los diferentes niveles de uso del informe de auditoría.

Palabras clave: Diferencias de expectativas en auditoría; Informe de los auditores; Nuevo informe de auditoría

\section{Introdução}

A auditoria independente tem a função de atestar a confiabilidade das informações financeiras das entidades (Mafra, Cavalcanti \& Penha, 2017). No advento da profissão, auditores eram contratados para examinar todas as transações das companhias e opinar com precisão sobre a existência ou não de fraudes (Boyd, Boyd \& Boyd, 2001). Com o crescimento das empresas e surgimento de operações cada vez mais complexas, como transações envolvendo leasing e derivativos, os exames dos auditores passaram a ser realizados por amostragens e opiniões emitidas com objetivo de assegurar a razoabilidade das demonstrações financeiras, de acordo com seus aspectos relevantes (Fonseca, Nunes \& Santana, 2016).

Apesar da adaptação do papel do auditor ao contexto globalizado, ainda persistem litígios entre sociedade e auditores, amplificados por escândalos financeiros abarcando companhias brasileiras de grande porte, como Sadia, Aracruz, Banco Santos, Banco Panamericano e, mais recentemente, Petrobras, Odebrecht e J\&F (Castro, Amaral \& Guerreiro, 2019). Os escândalos evidenciam que os usuários das demonstrações contábeis podem esperar resultados diferentes dos trabalhos dos auditores em comparação ao que estes realizam (Almeida, 2004). Esses descompassos destacaram as discussões acerca da integridade das atividades do auditor independente e da eficácia do relatório de auditoria (Mock, Bédard, Coram, Davis, Espahbodi \& Warne, 2013).

Divergências nas expectativas em relação aos deveres dos auditores, entre auditores e demais usuários das demonstrações contábeis, caracterizam a denominada Audit Expectation Gap (AEG), traduzidas como Diferenças de Expectativas em Auditoria (DEA) (Sterzeck, 2017). Sua composição pode ser desmembrada pelas seguintes parcelas:

- Diferença de Razoabilidade - descompasso entre as responsabilidades que a sociedade espera dos auditores e o que é razoável esperar pelos seus trabalhos de acordo com as normas e leis;

- Padrões Deficientes - diferença entre as responsabilidades razoáveis esperadas pela sociedade e as responsabilidades determinadas pelas normas;

- Desempenho Deficiente - diferença entre o desempenho que o auditor deveria atender, de acordo com as normas, e o desempenho percebido pela sociedade (Porter, 1993).

No conjunto das diferenças de expectativas em auditoria, existem também lacunas de informação e comunicação entre os auditores e não-auditores (demais usuários do relatório) (Turner et al., 2010; IAASB; 2011; Mock et al., 2013; Litjens, Buuren \& Vergoossen, 2015). A lacuna de informação pode ser compreendida como a diferença entre o que os não-auditores desejam receber de informação e o que está disponível para eles através do relatório (IAASB, 2011), enquanto a lacuna de comunicação representa a diferença entre o que os não-auditores compreendem e o que é comunicado pelos auditores (Mock et al., 2013).

Devido a constante insatisfação do mercado em relação ao desempenho dos auditores (Cordos \& Fulop, 2015; Defond \& Zhang, 2014; Kamau, 2013; Salehi, 2011), ampliada por sucessivos escândalos financeiros (Enron, 2001; Worldcom, 2002; Xerox, 2002) em conjunto com a percepção dos padrões deficientes (Porter \& Gowthorpe, 2004) foram conduzidas revisões das Normas Internacionais de Auditoria (ISAs) para atingir, de forma geral, maior transparência e objetividade. De acordo com o IAASB, as revisões seguiam os seguintes propósitos: identificar e esclarecer nas ISAs os propósitos gerais do auditor; estipular um objetivo para cada ISA e as obrigações do auditor com relação a cada objetivo; esclarecer as obrigações impostas ao auditor e a linguagem utilizada; reduzir a possibilidade de ambiguidade sobre as exigências do auditor a serem atingidas; melhorar a leitura e entendimento sobre as ISAs. A minuta de exposição das novas normas de relatório foi apresentada em 25 de julho de 2013, no documento denominado "Reporting on Audited Financial Statements: Proposed New e Revised International Standards on Auditing" (IAASB, 2013). Em 2017, as novas normas do Relatório dos Auditores no Brasil foram implantadas. 
A possível existência de DEA em relação aos deveres da auditoria externa é o fato motivador para a realização desta pesquisa. A presença destas diferenças alimenta o ambiente de críticas e litígios contra a integridade dos auditores, podendo causar o rompimento de confiança entre estes e os demais usuários das demonstrações financeiras (Almeida, 2004). Caso as DEA sejam acentuadas, a confiança no relatório de auditoria é reduzida, o que, consequentemente, pode dificultar o processo de tomada de decisões dos investidores e demais stakeholders (Asare \& Wright, 2012). Diante do exposto, o presente trabalho visa avaliar a seguinte questão de pesquisa: Qual a diferença entre as expectativas de auditores e da sociedade, após a atualização das normas de auditoria ocorrida em 2017, no Brasil?

Partindo da questão de pesquisa estabelecida pelo trabalho, pode-se definir como objetivo geral da pesquisa verficar a existência de diferenças de expectativa em auditoria entre os auditores e não-auditores, além de detectar possíveis fatores que afetam a diferença, tais como o nível de utilização do relatório e o gênero do respondente.

Destaca-se que a auditoria, em geral, trata-se de uma atividade que absorve um elevado número de profissionais contábeis e ainda conta com lacunas de pesquisas acadêmicas (Sterzeck, 2017). No Brasil, os estudos de Almeida (2004), Albuquerque (2009), Menezes e Costa (2012), Oliveira (2015) e Wanderley (2017) trouxeram importantes contribuições em relação à existência das DEA. Sterzeck (2017) identificou a existência do DEA na razoabilidade nas decisões de litígios nos quais as empresas de auditoria figuram como polo passivo; enquanto Santos e Caldas (2019) encontraram diferença de expectativa na auditoria governamental entre os grupos pesquisados. O presente estudo visa contribuir nessa ceara de pesquisa, após a implantação do formato atualizado do relatório do auditor.

A possível existência de uma discrepância entre as expectativas dos auditores e dos demais usuários em relação aos deveres dos auditores é o fato motivador para a realização desta pesquisa. A presença destas diferenças alimenta o ambiente de crítica e litígio contra a integridade dos auditores, podendo causar o rompimento de confiança entre estes e os demais usuários das demonstrações financeiras (Almeida, 2004). A investigação do tema é relevante não apenas aos auditores, órgãos governamentais e demais usuários da contabilidade, como também para toda sociedade em geral.

Por parte dos auditores, a investigação das DEA é relevante pois impacta a segurança jurídica desses profissionais e também evidencia uma discussão sobre seu papel perante os usuários do relatório do auditor e seu papel para com a sociedade (Sterzeck, 2017). Para os órgãos reguladores, a pesquisa na área é constituída de uma contribuição teórica, podendo ser utilizada para embasamento de futuros estudos e para formulação e implementação de normas e medidas preventivas. Aos demais usuários e sociedade, em geral, a exploração do tema contribui para o melhor entendimento dos deveres dos auditores e dos limites do relatório da auditoria, podendo, assim, trazer mais segurança na avaliação econômico-financeira das instituições, detectando riscos relacionados e, inclusive, auxiliando na escolha de possíveis investimentos futuros.

\section{Revisão de Literatura}

\subsection{Novo Relatório do Auditor}

Após a promulgação das normas internacionais de auditoria (ISAs) no ano de 2015, o Brasil iniciou o procedimento de tradução integral e catalogação no contexto brasileiro. Posto isso, o Comitê de Pronunciamentos Contábeis (CPC), a Comissão de Valores Mobiliários (CVM) e o Conselho Federal de Contabilidade (CFC) ofereceram aos usuários, por meio de edital de audiência pública, as minutas traduzidas para efeito de debates, comentários e estudos. Após este procedimento, as normas foram aprovadas e publicadas, em junho de 2016, no Diário Oficial da União (DOU), com vigência para auditorias de demonstrações contábeis de períodos que se findam em (ou após) 31 de dezembro de 2016. Destaca-se que a classificação e denominação seguiram os padrões internacionais, conforme ilustrado na Figura 1.

\begin{tabular}{|l|l|l|}
\hline \multicolumn{1}{|c|}{ NBC } & \multicolumn{1}{c|}{ Nome da Norma } & \multicolumn{1}{c|}{ IFAC } \\
\hline NBC TA 260 (R2) & Comunicação com os Responsáveis pela Governança & ISA 260 \\
\hline NBC TA 570 & Continuidade Operacional & $\begin{array}{l}\text { Formação da Opinião e Emissão do Relatório do Auditor Independente sobre } \\
\text { as Demonstrações Contábeis; }\end{array}$ \\
\hline NBC TA700 & $\begin{array}{l}\text { Comunicação dos Principais Assuntos de Auditoria no Relatório do Auditor } \\
\text { Independente; }\end{array}$ & ISA 700 \\
\hline NBC TA 701 & Modificações na Opinião do Auditor Independente; & ISA 705 \\
\hline NBC TA 705 & $\begin{array}{l}\text { Parágrafos de Ênfase e Parágrafos de Outros Assuntos no Relatório do } \\
\text { Auditor Independente. }\end{array}$ & ISA 706 \\
\hline NBC TA 706 &
\end{tabular}

Figura 1 - Correlação entre normas Nacionais e Internacionais

Fonte: Site do Conselho Federal de Contabilidade - CFC, 2016

Dada a aprovação e divulgação das normas, cabe avaliar os seus efeitos. De acordo com Manoel e Quel (2017), dentre as modificações fundamentais, ocorridas no intuito de atender às expectativas do usuário, 
estão: a reorganização dos parágrafos; a confirmação da independência do auditor; comentário dos auditores e dos executivos em relação às possíveis incertezas da continuidade operacional da entidade; inclusão do Key Audit Matters ou Principais Assuntos de Auditoria; Adição de seção sobre outras informações acerca das demonstrações contábeis; e descrição mais detalhada sobre as responsabilidades do auditor.

A primeira mudança estrutural, citada por Manoel e Quel (2017), corresponde à reorganização dos parágrafos do relatório do auditor. Destaca-se que a opinião do auditor, antes apresentada nas partes finais do relatório, passa para o início e incorpora o parágrafo introdutório. Em seguida, abaixo da opinião, apresenta-se a base da opinião. De acordo com Veiga, Ribeiro e Inácio (2017), nesta seção o auditor deve mencionar, dentre outras informações, o motivo que levou à modificação da opinião e se a auditoria foi realizada de acordo com as normas e requisitos éticos.

Outro ponto de destaque é referente ao realce da questão sobre a continuidade operacional. De acordo com a revisão realizada na ISA 570, o relatório passa a incluir informações sobre a responsabilidade do auditor e da gestão referente às incertezas no tocante à atividade (continuidade) da empresa. Desta forma, há a inserção do tópico de incertezas relevantes.

Para as empresas listadas em bolsa de valores, torna-se obrigatório a evidenciação dos Key Audit Matters (KAM), traduzidos como Principais Assuntos de Auditoria (PAA), e a divulgação do nome do sócio responsável pelos trabalhos. De acordo com a NBC TA 701, item 8, os Principais Assuntos de Auditoria (PAA) são assuntos que foram os mais relevantes no processo de auditoria das demonstrações contábeis do período. Destaca-se, desta forma, que os principais assuntos de auditoria são selecionados entre os assuntos comunicados aos responsáveis pela governança.

Cordos e Fulop (2015, p.132) sublinham que "a comunicação de KAMs exige que o auditor aplique seu julgamento profissional, mas também tenha em conta a natureza e extensão da comunicação com os responsáveis pela governança". Nesse sentido, os principais assuntos de auditoria representam a principal mudança no relatório dos auditores (Bédard \& Gonthier-Besacier; Schatt, 2014; Cordos \& Fulopa, 2015).

Vale observar que as mudanças ocorridas no relatório dos auditores não esgotam a possibilidade de futuras alterações no formato e na escrita, além de inclusões de novas informações, com o objetivo de redução das diferenças de expectativas em auditoria. Pesquisas são desenvolvidas para avaliar as possíveis inclusões de informações e alterações do relatório, as quais serão abordadas no próximo tópico.

\subsection{Diferenças de Expectativas em Auditoria}

A fonte do problema das diferenças de expectativas é apresentada sob duas visões por Porter (1993). A primeira visão atribui a manutenção das DEA à ignorância da sociedade a respeito da natureza dos trabalhos da auditoria. Este ponto de vista tem sido utilizado pelos profissionais de auditoria para contrariar às críticas no seu desempenho (Manson \& Zamon, 2001). Consequentemente, as estratégias adotadas pelos profissionais de auditoria ao longo dos anos para superar as DEA têm sido orientadas no sentido de educar os usuários das demonstrações contábeis e aumentar a consciência da sociedade sobre o significado de uma auditoria (Maseko, 2016). Esses esforços incluem a publicação de normas de auditoria e distribuição de panfletos educacionais (Geiger, 1994).

Porter (1993) segregou as DEA em dois componentes estruturais, sendo: diferença de razoabilidade e diferença de desempenho. De acordo com a autora, a diferença de razoabilidade corresponde ao gap entre as responsabilidades que a sociedade espera dos auditores e o que é razoável de se esperar pelos seus trabalhos. A Diferença de Desempenho, que é subdividida em Padrões Deficientes e Desempenho Deficiente, é caracterizada como a lacuna entre a responsabilidade que a sociedade espera dos auditores e a respectiva percepção da sociedade. Os Padrões Deficientes representam o gap entre as responsabilidades esperadas pelos usuários e as responsabilidades determinadas por normas e leis. O Desempenho Deficiente equivale à diferença entre o desempenho que o auditor deveria atender de acordo com as normas e leis versus o desempenho percebido pela sociedade. Ilustrativamente, os componentes podem ser representados da seguinte forma, conforme apresentado na Figura 2.

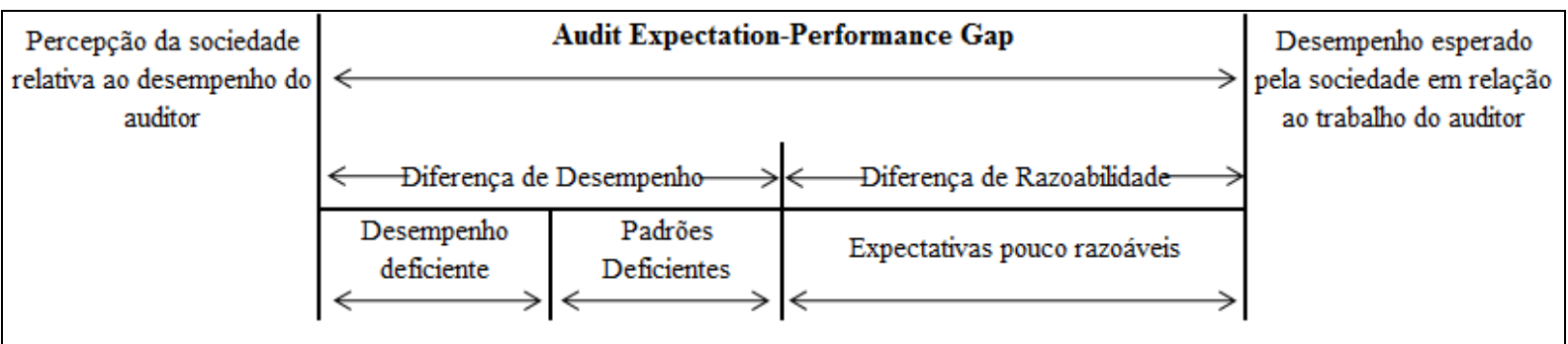

Figura 2 - Componentes estruturais do DEA

Fonte: Adaptado de Almeida, 2004

Conforme demonstrado por Almeida (2004), a partir dessa estrutura das diferenças de expectativas, destaca-se que a fronteira entre a diferença de razoabilidade e diferença de desempenho é constituída pelos 
deveres que se pode razoavelmente esperar dos auditores. Os componentes esclarecendo que a diferença de razoabilidade ocorre devido aos usuários esperarem mais que a auditoria pode oferecer, por exemplo, no caso em que o público espera que o auditor detecte integralmente as fraudes, independentemente de sua materialidade e relevância. Para as normas inadequadas, Almeida (2004) explica que é o caso de o usuário esperar que o auditor informe desvios de fundos às entidades reguladoras enquanto as leis ou regulamentos não o exijam. O desempenho inadequado, por sua vez, seria o fato de o auditor não mencionar dúvidas que existam sobre a continuidade da empresa, procedimento este estabelecido pelas normas.

Füredi-Fülöp (2015), em estudo realizado na Hungria, constatou que as DEA decorrem da combinação do desempenho deficiente dos auditores, deficiências nos padrões de auditoria, expectativas desproporcionais e interpretações erradas das funções de auditoria. Veiga et al. (2017) apontam que as normas inadequadas se referem à forma como as normas e regulamentos profissionais condicionam a função da auditoria e o seu papel, enquanto o desempenho inadequado está relacionado à falta de zelo profissional e competência técnica exigível do auditor. Para efeito de análise, Porter (1993) levantou, em sua pesquisa, a representatividade de cada componente na construção das DEA, detectando, naquela oportunidade, que: $16 \%$ referiam ao desempenho inadequado; $34 \%$ era dado pelas diferenças de razoabilidade e $50 \%$ dado pelas normas inadequadas.

Porter e Gowthorpe (2004) replicaram o estudo de Porter (1993), com o objetivo de avaliar a composição das DEA no Reino Unido, em comparação com a Nova Zelândia. O estudo constatou que, diferentemente da Nova Zelândia, o Reino unido apresentou uma maior proporção nas diferenças de razoabilidade (50\%). Estudos realizados na última década têm apontado para esta mudança, no qual o componente de maior representatividade na composição das DEA é dado pelas expectativas pouco razoáveis dos usuários (Almeida \& Colomina, 2008; Salehi, Mansoury \& Azary, 2009; Gold, Gronewold \& Pott, 2012; Kamau, 2013).

A composição das DEA em componentes estruturais facilitou a identificação e diferenciação das principais causas das diferenças de expectativas. Nesse sentido, delimitar e pormenorizar as possíveis causas inerentes à cada tipo de diferença colabora com a busca por estratégias eficazes para a redução das DEA. Dutra (2011) explica que a divisão em componentes facilita a obtenção de informações sobre cada item e colabora para concentração de esforços na tentativa de reduzir os gaps existentes.

Gray et al. (2011) ampliaram os trabalhos sobre as razões determinantes para o desempenho inadequado, normas inadequadas e expectativas pouco razoáveis. Neste contexto, os autores relacionam a falta de competência com a falta de conhecimento e de experiência, seja devido à ignorância dos auditores em relação à suas atribuições ou então devido à complexidade dos negócios. Quanto à independência do auditor, os autores destacam a importância do acesso à todas as informações e à necessidade de que não sejam realizadas modificações dos relatórios, devido pressões de diretores das empresas auditadas. A independência da profissão é relacionada à ausência (ou presença tardia) de normas com maior rigor aos profissionais e à concentração das empresas de auditoria. A clarificação do papel do auditor retrata que se as atribuições do auditor forem esclarecidas, logo as expectativas dos usuários tendem a se tornar razoáveis.

As diferenças de expectativas foram confirmadas em diferentes países e em diferentes períodos de tempo, como por Sule, Yusof e Bahador (2019). Os autores aplicaram questionários entre acadêmicos, contadores, investidores, corretores de bolsa e banqueiros. Os resultados reforçaram as diferenças de expectativas na área de prevenção e detecção de fraude, na Nigéria.

No Vietnam, Dung e Dang (2019) coletaram dados de (i) auditores (ii) auditados, (iii) usuários de demonstrações financeiras auditadas (iv) público em geral. O número de respostas alcançadas foi de 454 ou $32 \%$ do envio total de questionários. Os resultados explicitaram a presença das DEA nas três esferas apontadas por Porter (1993): (i) gap de razoabilidade; (ii) gap de desempenho dos auditores e (iii) gap de padrões.

Akther, Fengju e Haque (2019) avaliaram as percepções de investidores do mercado de capitais que movimentam títulos na bolsa de valores de Dhaka. O grupo de auditores foi selecionado aleatoriamente dentre auditores pertencentes à associação de classe do país. A amostra final foi composta por 130 respondentes. Os autores concluíram que, em Bangladesh, maior parte da lacuna foi apontada como lacuna de razoabilidade, que se encontra entre as responsabilidades que a sociedade espera dos auditores e o que é razoável esperar pelos seus trabalhos segundo normas e leis.

No Brasil, Wanderley (2017) identificou que as evidências obtidas sugerem que as diferenças de expectativas também sejam percebidas no país e decorrem, principalmente, de expectativas não razoáveis dos usuários das demonstrações contábeis e de uma maior demanda por informações em relação ao trabalho de auditoria.

\subsection{Impacto do Novo Relatório do Auditor nas Diferenças de Expectativas}

No contexto da adoção do novo relatório dos auditores, Gold et al. (2012) avaliaram o estado das diferenças de expectativas em auditoria na Alemanha e testaram se a presença de novas informações nos relatórios ocasionaria uma redução nas diferenças de expectativas em auditoria. Questionários foram enviados a auditores de firmas Big Four, analistas financeiros e estudantes. Os achados sugeriram que as 
diferenças de expectativas na auditoria persistem no cenário germânico, corroborando com os estudos de Best, Buckby e Tan (2001). Adicionalmente, os autores constataram que as modificações apresentadas no novo relatório do auditor não afetaram as diferenças de expectativas em auditoria.

Breesch, Hardies e Muylder (2012) realizaram um experimento para identificar qual dos processos é mais eficaz na redução das DEA: a expansão do relatório do auditor ou a educação dos usuários. O estudo foi conduzido com 159 estudantes de economia da Bélgica. Os resultados apontaram que a educação em auditoria reduz significativamente as DEA dado que, de acordo com as análises, os participantes das aulas de auditoria tiveram um entendimento mais apropriado da função e das responsabilidades do auditor em relação à prevenção e detecção de fraude e referente à avaliação da continuidade da empresa. Adicionalmente, os autores constataram, assim como Gold et al. (2012), que o relatório expandido dos auditores não afeta significativamente a compreensão dos usuários sobre auditoria, de forma geral, mas apenas em pontos isolados como: detecção de fraude, segurança e amostragem.

Mock et al. (2013) realizaram um levantamento de pesquisas publicadas no período entre 2006 e 2011, no intuito de identificar quais tipos de informações específicas os stakeholders desejavam incluir no relatório de auditoria. Os achados demonstraram que informações relacionadas ao processo de auditoria, independência do auditor, materialidade, nível de garantia que o auditor está fornecendo, informações referentes à entidade, políticas contábeis e riscos de continuidade foram os tópicos mais recorrentes. Ademais, os autores também destacaram que alterações do relatório no sentido de explicar melhor as responsabilidades dos auditores e da administração foram notadas pelos stakeholders, entretanto, não pareceram melhorar o valor comunicativo do relatório de auditoria e contribuir para redução das diferenças de expectativas.

As informações incluídas no novo relatório do auditor, o confronto entre qualidade ou quantidade de informações e seus efeitos nas diferenças de expectativas em auditoria foram foco do estudo de Litjens et al. (2015). Os autores realizaram uma pesquisa na Holanda avaliando o efeito da inclusão de informações sobre a empresa auditada, sobre o processo de auditoria e sobre a modificação do relatório dos auditores nas DEA. Dado o objetivo proposto, 1.520 questionários construídos com base na escala Likert (com intervalos entre 0 a 6) foram enviados a uma população composta por: gestores de empresas privadas holandesas (gerenteproprietário, diretor-presidente, diretor financeiro) que necessitam de auditoria obrigatória; banqueiros que operam no mercado de empréstimos de empresas privadas; e auditores de firmas de auditoria de médio porte. Obteve-se uma amostra final de 302 respondentes (123 auditores, 118 gestores e 61 bancários), totalizando, aproximadamente, $20 \%$ da população. Os resultados, que reforçaram os achados de Gold et al. (2012), apontaram que os banqueiros não atribuiram alto valor às mudanças no formato e escrita no relatório do auditor, e que estas mudanças não afetaram as diferenças de expectativas. Por outro lado, os autores destacaram que informações sobre o processo de auditoria relacionados à continuidade e evidenciação dos erros são capazes de reduzir as diferenças de expectativas dos bancários.

Boolaky e Quick (2016) investigaram o impacto da divulgação do nível de segurança, materialidade e pontos-chave da auditoria no novo relatório do auditor, por meio da percepção dos diretores bancários alemães sobre a qualidade das demonstrações financeiras, processos de auditoria e do relatório de auditoria para decisão de aprovações de crédito. A amostra foi composta por 105 diretores bancários, e a análise de Covariância (ANCOVA) foi utilizada para determinar as variáveis que explicam as percepções e decisões dos diretores. Os resultados sugeriram que a divulgação do nível de segurança teve um impacto positivo nas decisões para concessão de crédito, enquanto a divulgação da materialidade e pontos-chave de auditoria não alteraram significativamente as percepções dos diretores de instituições financeiras.

Lundgren e OldenBorg (2016) desenvolveram uma pesquisa, na Suíça, com objetivo de analisar como a adoção das normas revisadas de auditoria (ISA 700 e ISA 701) impactou na comunicação, no expectation gap e na atribuição de valor ao relatório do auditor, por parte dos investidores não-profissionais suíços. Os resultados apontaram que $64 \%$ dos investidores consideraram o novo relatório mais informativo e que as mudanças, dadas principalmente pela inclusão dos pontos-chave de auditoria, aumentaram o potencial de possíveis leitores do relatório dos auditores.

Maseko (2016) pesquisou se o novo relatório do auditor, dado pela revisão da norma ISA 700, realizado pelo IAASB, reduziria as diferenças de expectativas em auditoria no contexto da África do Sul. O estudo focou nas diferenças de expectativas referentes à responsabilidade dos auditores, fidedignidades das demonstrações financeiras auditadas e utilidade das demonstrações auditadas para tomada de decisão. Uma amostra de 300 participantes (100 auditores Big 4, 100 bancários e 100 estudantes), obtida através da rede Linkedin, foi selecionada. Os testes não paramétricos de Kruskal-Wallis e Mann-Whitney foram utilizados para analisar os dados. Os resultados mostraram que, em geral, apesar das modificações no relatório de auditoria, as diferenças de expectativas mantiveram-se em relação às responsabilidades dos auditores. Destacou-se, assim como Lundgren e Oldenborg (2016), que o novo relatório aumentou a confiança dos usuários nas demonstrações contábeis e a sua utilidade como instrumento de tomada de decisões.

Em Portugal, Veiga et a. (2017), ao aplicarem questionários e entrevistas a analistas financeiros, auditores e gestores, encontraram evidências de que, na percepção dos inquiridos, as alterações no relatório apresentaram efeito positivo na redução das expectativas em auditoria no país. Em termos práticos, avaliando especificamente como empresas de auditoria lidam com os requisitos da ISA 701 em relação aos relatos de 
níveis de materialidade, Iwanowicz e Iwanowicz (2019) analisaram empresas listadas na Bolsa de Varsóvia (158 empresas) e Londres (159 empresas). A partir da análise empírica, concluiu-se que a implementação da ISA 701 e a divulgação da materialidade limitaram a lacuna das expectativas da auditoria.

Iwanowicz e Iwanowicz (2019) e Coram e Wang (2020) analisaram um dos pontos específicos das alterações no Relatório do Auditor Independente: a Comunicação dos Principais Assuntos de Auditoria, considerado um dos pontos mais relevantes no processo de auditoria das demonstrações contábeis. Os autores perceberam que a divulgação dos principais assuntos, por si só, no relatório de auditoria, não afetou a lacuna das expectativas de auditoria.

Dessa forma, verifica-se que, em geral, os resultados não apresentam consenso sobre o efeito da adoção das revisões das normas internacionais de auditoria nas diferenças de expectativas dos usuários. Nwaobia e Theophilus (2016) defendem que os novos padrões de relatórios dos auditores propiciam impactos positivos no longo prazo, no sentido de limitar as diferenças de expectativas. Contudo, os resultados somente seriam alcançados com o atendimento total da norma. Para tal, os auditores e os órgãos profissionais de contabilidade precisariam assumir maior responsabilidade, por meio de sistemas de controle de qualidade aprimorados e equipes com um engajamento ético, comprometidas com a qualidade.

\section{Abordagem Metodológica}

\subsection{População e Amostra}

A população alvo da análise corresponde a todos os profissionais que atuam como auditores independentes e todos os profissionais que utilizam as demonstrações contábeis auditadas para a tomada de decisões. A amostra foi constituída por conveniência, de acordo com a disponibilidade de respondentes para a análise. Diante de uma amostra selecionada de forma não aleatória, observa-se limitações na capacidade de generalização dos resultados encontrados.

A amostra compreendeu profissionais que atuam como auditores independentes em firmas, ou autônomos, e não-auditores. No grupo dos auditores, foram convidados a participar todos os profissionais da área, independente do cargo exercido. Para o grupo dos não-auditores foram selecionados respondentes envolvidos com contabilidade ou profissionais que utilizam as demonstrações contábeis auditadas para a tomada de decisões. A amostra não probabilística contou com 185 respostas completas e o instrumento foi aplicado em junho e julho de 2017. Os instrumentos utilizados foram idênticos nos dois grupos de respondentes (auditores e não-auditores).

\subsection{Escalas propostas e validação}

Este estudo fez uso de um questionário baseado no instrumento utilizado na pesquisa de Litjens et al. (2015). O instrumento de coleta de dados foi validado por quatro professores com experiência em contabilidade e auditoria. Os especialistas geraram 25 contribuições de ordem técnica, semântica e de conteúdo. Para a construção das escalas, optou-se por utilizar sete escores, sendo que a escala mínima correspondeu a "Não melhora a compreensão do papel da auditoria" (pontuação igual a 1) e a escala máxima correspondeu a "Melhora fortemente a compreensão do papel da auditoria" (pontuação igual a 7). A distribuição das sentenças foi a seguinte: 12 questões sobre o perfil do respondente e 18 para avaliação das expectativas em relação aos deveres do auditor contábil. Os instrumentos utilizados foram idênticos nos dois grupos de respondentes (auditores e não-auditores).

A aplicação do instrumento utilizado na pesquisa de Litjens et al. (2015) foi autorizada pelos autores, não houve alteração substancial no instrumento de referência e todas as exigências de pesquisas com seres humanos foram respeitadas. Quanto ao quesito da confiabilidade, utilizou-se a medida do Alfa de Cronbach, sendo considerado o nível de confiabilidade mínimo de 0,7. Apresenta-se a seguir a distribuição das escalas por seções, sendo segregadas em perfil dos respondentes, mensuração das expectativas em relação aos deveres dos auditores e mensuração de importância atribuída em relação à inclusão de informações no relatório dos auditores.

\section{a) Mensuração das expectativas dos respondentes em relação aos deveres dos auditores}

Baseada nos constructos de pesquisa utilizados por Litjens et al. (2015), embasados no trabalho de Porter (1993), 18 sentenças foram definidas para mensurar o índice de expectativa dos auditores e não auditores. Destaca-se que o Índice de Expectativas, nesse sentido, é constituído de três partes, sendo: Desempenho Deficiente - DD (sentenças 1 a 6), Padrões Deficientes - PD (sentenças 7 a 11) e Diferença de Razoabilidade - DR (sentenças 12 a 18), conforme apresentado na Figura 3.

As declarações que visam mensurar o índice de expectativas são segregadas em: deveres existentes dos auditores, deveres razoavelmente esperados dos auditores e deveres que não podem ser esperados dos auditores. 
Para a presente pesquisa, seguindo o método adotado por Hassink, Bollen, Meuwissen, \& Vries (2009) e Litjens et al. (2015), os respondentes apontaram, segundo o próprio julgamento, o grau de concordância ou discordância em relação às sentenças propostas com base na escala Likert. Os itens componentes do índice são apresentados na Figura 3.

\begin{tabular}{|l|c|}
\hline \multicolumn{1}{|c|}{ Em que medida você acredita que o respectivo item é um dever relevante de um auditor independente? } \\
\hline \multicolumn{1}{|c|}{} \\
\hline 1. O auditor deve ser independente. & GD \\
\hline 2. O auditor deve declarar se as demonstrações financeiras refletem adequadamente os negócios de uma empresa. \\
\hline 3. O auditor contábil deve auditar apenas as demonstrações financeiras. \\
\hline $\begin{array}{l}\text { 4. O auditor deve detectar e divulgar distorções intencionais de informações financeiras que têm impacto relevante nas } \\
\text { demonstrações financeiras. }\end{array}$ \\
\hline 5. O auditor deve detectar roubo de ativos corporativos pelos funcionários e pela administração. \\
\hline 6. O auditor deve assegurar o cumprimento das normas contábeis. \\
\hline $\begin{array}{l}\text { 7. O auditor deve examinar e reportar aos usuários a fidedignidade das estimativas financeiras apresentadas pela } \\
\text { empresa nas demonstrações. }\end{array}$ \\
\hline 8. O auditor deve relatar suspeitas de fraude aos órgãos reguladores. \\
\hline 9. O auditor deve relatar aos órgãos reguladores as distorções intencionais de informações. \\
\hline 10. O auditor deve avaliar e relatar a qualidade dos controles internos. \\
\hline 11. O auditor deve avaliar e relatar como os riscos de fraude são gerenciados. \\
\hline 12. O auditor deve elaborar as demonstrações financeiras. \\
\hline 13. O auditor deve detectar e relatar fraudes que não afetam diretamente as contas da empresa. \\
\hline 14. O auditor deve verificar/analisar todas as transações contábeis ocorridas no exercício contábil. \\
\hline 15. O auditor deve verificar o pressuposto da continuidade em relação às atividades da empresa. \\
\hline 16. O auditor deve garantir que as demonstrações financeiras sejam precisas. \\
\hline 17. O auditor deve examinar e relatar a eficácia/eficiência da gestão. \\
\hline 18. O auditor deve revelar distorções intencionais de ativos corporativos por funcionários. \\
\hline
\end{tabular}

Figura 3 - Expectativas em relação aos deveres dos auditores

Nota: Desempenho Deficiente, PD = Padrões Deficientes; $D R=$ Diferença de razoabilidade .

Fonte: Baseado nos trabalhos de Litjens, Buuren e Vergoossen (2015)

Posteriormente, o índice de expectativa de cada respondente foi calculado levando-se em consideração a média dos componentes das DEA:

\section{b) Perfil dos Respondentes}

$$
\mathrm{IE}=\frac{\sum(\mathrm{DD}, \mathrm{PD}, \mathrm{DR})}{\mathrm{n} \text { o sentenças }}
$$

Para avaliar o perfil dos respondentes foram elaboradas questões que captaram características pessoais como: gênero, idade, grau de instrução, área de formação, profissão atual, área que desenvolve as atividades, tempo de experiência na profissão atual e cargo atual. Adicionalmente, foram elencadas, também, questões relacionadas ao envolvimento do respondente com auditoria e com o relatório dos auditores. Para tanto, questionou-se o tipo de experiência com auditoria (apenas acadêmica, acadêmica e profissional, apenas profissional ou nenhuma), se o inquerido leu algum relatório do auditor publicado em 2017, e, por fim, se considera que o relatório de auditoria, como meio de comunicação, pode ser melhorado.

\subsection{Teste de Média}

A presente pesquisa fez o uso de testes de Mann-Whitney para verificar a existência de diferenças estatisticamente significativa de expectativas entre grupos de usuários do relatório do auditor. O teste de MannWhitney constitui-se como uma alternativa não-paramétricas dos testes de média para amostras independentes. O teste de Mann-Whitney é um teste estatístico que verifica a existência de diferenças significativas entre a soma dos postos de duas amostras. As hipóteses do teste de Mann-Whitney são estabelecidas em termos dos valores medianos da amostra estudada (Siegel \& Castellan Jr., 2006).

\section{Discussão dos Resultados}

\subsection{Estatisticas Descritivas}

A amostra inicialmente coletada foi constituída por 194 questionários respondidos, dentre os quais 9 apresentaram dados faltantes (missing data), sendo consideradas para fins de análise, portanto, um total de 185 observações, constituídas por 40 auditores e 145 não-auditores (demais usuários das informações contábeis).

Antes de abordar aspectos referentes às expectativas dos respondentes acerca dos deveres dos auditores, apresenta-se as principais características amostrais, tais como gênero, grau de instrução, área de formação, profissão, entre outras. 
No que se refere ao gênero, constata-se a preponderância de respondentes do gênero masculino comparada ao gênero feminino, na amostra coletada. $\mathrm{Na}$ análise em conjunto (auditores e não-auditores), dentre os 185 respondentes, $44 \%$ assinalaram ser do gênero feminino, correspondendo a 82 observações, e $56 \%$ assinalaram ser do gênero masculino, correspondendo a 103 observações. Ao analisar separadamente, por grupo de usuários, observou-se que o número do gênero masculino é dado em maior escala no grupo dos respondentes que trabalham com auditoria, sendo que, do total de 40 auditores, $68 \%$ assinalaram ser do gênero masculino, correspondendo 27 observações, e 33\% do gênero feminino, correspondendo a 13 observações.

Um dos fatores que explicam a prevalência do gênero masculino na amostra pode estar relacionado às diferenças entre os gêneros historicamente registrada no mercado de trabalho. Apesar de estar aumentando, a taxa de participação das mulheres no mercado de trabalho ainda está marcada por uma forte diferença em relação a dos homens (Abram, 2006). Entretanto, cabe destacar que a presença feminina no contexto do mercado de trabalho relacionado à contabilidade e auditoria tende a crescer, dado o grande número de estudantes desse gênero em formação (Cruz, Lima, Durso \& Cunha, 2018).

Outro ponto observado refere-se à faixa etária da amostra em estudo. De acordo com a Tabela 1, a maior concentração foi dada na faixa de 21 a 30 anos, representando $52 \%$ dos respondentes. A idade mínima da amostra foi de 19 anos e a máxima de 59. Destaca-se, também, que a idade de maior frequência foi de 28 anos. No que se refere à área de formação, é destacado o fato de que a maioria dos respondentes são formados em Ciências Contábeis, no total de 85\%, representando 157 respondentes. Em segundo, observase $8 \%$ dos respondentes formados em administração e 7\% formados em outras áreas, tais como Direito, Economia, Estatística, entre outros.

Tabela 1:

Perfil dos Respondentes

\begin{tabular}{|c|c|c|c|c|c|c|c|}
\hline \multicolumn{2}{|c|}{ Identificação } & \multirow{2}{*}{$\begin{array}{c}\begin{array}{c}\text { Freq. } \\
\text { Abs }\end{array} \\
82\end{array}$} & \multirow{2}{*}{$\begin{array}{c}\% \\
44.3 \%\end{array}$} & \multicolumn{2}{|c|}{ Identificação } & \multirow{2}{*}{$\begin{array}{c}\begin{array}{c}\text { Freq. } \\
\text { Abs }\end{array} \\
157 \\
\end{array}$} & \multirow{2}{*}{$\begin{array}{c}\% \\
84.9 \%\end{array}$} \\
\hline \multirow{3}{*}{ Gênero } & Feminino & & & \multirow{6}{*}{$\begin{array}{l}\text { Área de } \\
\text { Formação }\end{array}$} & Ciências & & \\
\hline & Masculino & 103 & $55.7 \%$ & & Administração & 15 & $8.1 \%$ \\
\hline & TOTAL & 185 & $100.0 \%$ & & Economia & 5 & $2.7 \%$ \\
\hline \multirow{6}{*}{ Faixa Etária } & Até 20 anos & 3 & $1.6 \%$ & & Direito & 4 & $2.2 \%$ \\
\hline & Entre 21 e 30 anos & 97 & $52.4 \%$ & & Outras & 4 & $2.2 \%$ \\
\hline & Entre 31 e 40 anos & 54 & $29.2 \%$ & & TOTAL & 185 & $100.0 \%$ \\
\hline & Entre 41 e 50 anos & 21 & $11.4 \%$ & \multirow{5}{*}{$\begin{array}{c}\text { Ocupação } \\
\text { atual }\end{array}$} & Contador (a) & 123 & $66.5 \%$ \\
\hline & Acima de 50 & 10 & $5.4 \%$ & & Auditor (a) & 40 & $21.6 \%$ \\
\hline & TOTAL & 185 & $100.0 \%$ & & Estudante & 14 & $7.6 \%$ \\
\hline \multirow{6}{*}{$\begin{array}{c}\text { Grau de } \\
\text { Instrução }\end{array}$} & Doutor & 4 & $2.2 \%$ & & Outros & 8 & $4.3 \%$ \\
\hline & Mestre & 27 & $14.6 \%$ & & TOTAL & 185 & $100.0 \%$ \\
\hline & Especialista & 64 & $34.6 \%$ & \multirow{4}{*}{$\begin{array}{c}\text { Tempo de } \\
\text { experiência } \\
\text { na Profissão } \\
\text { Atual }\end{array}$} & Até 5 anos & 97 & $52.4 \%$ \\
\hline & $\begin{array}{l}\text { Ensino superior } \\
\text { completo }\end{array}$ & 65 & $35.1 \%$ & & Entre 6 e 15 anos & 66 & $35.7 \%$ \\
\hline & Ensino médio/técnico & 25 & $13.5 \%$ & & Acima de 15 & 22 & $11.9 \%$ \\
\hline & TOTAL & 185 & $100.0 \%$ & & TOTAL & 185 & $100.0 \%$ \\
\hline
\end{tabular}

Ao analisar a ocupação exercida pelos respondentes, verifica-se que $92 \%$ da amostra exercem profissões no mercado de trabalho, correspondendo a 171 observações distribuídas entre Contador, Auditor, Professor, Advogado, Bancário, Minerador, Técnico de Informação, Vendedor e Engenheiro. Dentre as ocupações informadas pelos respondentes, aquelas que obtiveram a maior representatividade foram a de Contador (a), Auditor (a), Professor (a) e Estudante, com a representatividade de respectivamente 56\%, 22\%, $11 \%$ e $8 \%$ da amostra, correspondendo ao total de respectivamente 103, 40, 20 e 14 observações.

Após analisadas as principais características que compõem o perfil dos respondentes, buscou-se avaliar o envolvimento e conhecimento dos respondentes em relação aos trabalhos de auditoria e o respectivo relatório dos auditores, conforme dados apresentados na Tabela 2.

Observa-se que $88 \%$ da amostra já teve ou tem experiência com auditoria, correspondendo a 163 respondentes. Destes, $42 \%$ assinalaram ter experiência acadêmica e profissional, que corresponde a 78 respondentes, 36\% com experiência apenas acadêmica, representando 66 observações e 10\% com experiência apenas profissional. Apesar da representatividade de respondentes com experiência em auditoria, seja na forma acadêmica e/ou profissional, apenas $38 \%$ da amostra assinalou ter lido o novo relatório dos auditores. Por fim, quando questionados se o relatório do auditor ainda poderia ser melhorado, verificou-se que $74 \%$ apontaram pela possível melhoria, enquanto $25 \%$ não souberam e $2 \%$ afirmaram que não cabem melhorias. 
Tabela 2:

Envolvimento dos respondentes com auditoria e relatório dos auditores

\begin{tabular}{|c|c|c|c|}
\hline Identificação & & Frequência & $\%$ \\
\hline Experiência com Auditoria & $\begin{array}{l}\text { Acadêmica e Profissional } \\
\text { Apenas Acadêmica } \\
\text { Apenas Profissional } \\
\text { Nenhuma } \\
\text { TOTAL }\end{array}$ & $\begin{array}{c}78 \\
66 \\
19 \\
22 \\
\mathbf{1 8 5} \\
\end{array}$ & $\begin{array}{r}42 \% \\
36 \% \\
10 \% \\
12 \% \\
100 \% \\
\end{array}$ \\
\hline Como utiliza o relatório dos auditores? & $\begin{array}{l}\text { Leio } \\
\text { Não Leio } \\
\text { TOTAL }\end{array}$ & $\begin{array}{c}109 \\
76 \\
\mathbf{1 8 5}\end{array}$ & $\begin{array}{l}59 \% \\
41 \% \\
100 \%\end{array}$ \\
\hline Leu o novo relatório dos auditores? & $\begin{array}{l}\text { Sim } \\
\text { Não } \\
\text { TOTAL }\end{array}$ & $\begin{array}{c}70 \\
115 \\
\mathbf{1 8 5}\end{array}$ & $\begin{array}{r}38 \% \\
62 \% \\
100 \%\end{array}$ \\
\hline Relatório pode ser melhorado? & $\begin{array}{l}\text { Sim } \\
\text { Não } \\
\text { Não Sei } \\
\text { TOTAL }\end{array}$ & $\begin{array}{c}136 \\
3 \\
46 \\
185 \\
\end{array}$ & $\begin{array}{c}74 \% \\
2 \% \\
25 \% \\
100 \% \\
\end{array}$ \\
\hline
\end{tabular}

\subsection{Estatísticas Inferenciais}

Para verificar se existem diferenças de expectativa em auditoria entre os auditores e não-auditores, utilizou-se, então, o teste não paramétrico de Mann-Whitney, cujos resultados são apresentados na Tabela 3.

Tabela 3:

Resultado do teste de Mann-Whitney para diferenças de expectativas em auditoria

\begin{tabular}{|c|c|c|c|}
\hline Grupo & Soma dos Postos & Valor Esperado & Valor-P \\
\hline Não-Auditores & 14.525 & 13.485 & \\
\hline $\begin{array}{l}\text { Auditores } \\
\text { Total }\end{array}$ & $\begin{array}{r}2.680 \\
17.205\end{array}$ & $\begin{array}{r}3.720 \\
17.205\end{array}$ & 0.0005 \\
\hline
\end{tabular}

Fonte: Dados da pesquisa

O resultado do teste apresentado na Tabela 3 aponta que a soma dos postos do grupo de auditores alcança um total de 2.680, inferior ao valor esperado de 3.720. Por outro lado, verifica-se que a soma dos postos dos não auditores, dada no total de 14.525, foi superior ao valor esperado, de 13.485. Neste rumo, ao analisar o valor-p do teste de Mann-Whitney, constata-se que houve a rejeição do pressuposto de comportamento igual dos Índices de Expectativa entre os grupos. Os achados seguem os estudos Best et al. (2001), Hassink et al. (2009), Albuquerque (2009) e Litjens et al. (2015).

Os principais motivos do distanciamento entre as expectativas dos auditores e não-auditores podem ser relativos a assuntos específicos, como a responsabilidade dos auditores na prevenção de fraudes, manutenção dos registros contábeis e julgamento na seleção dos procedimentos de auditoria (Best et al. 2001), temas nos quais os não-auditores podem criar expectativas superestimadas na tentativa de salvaguardar seus interesses. Outro ponto de destaque é que, apesar da recente adoção das normas internacionais de auditoria (ISAs 700, 701, 570, 705, 706, 260 e 720), que modificaram o relatório dos auditores, o gap entre a expectativa dos auditores e não-auditores permaneceu, no contexto brasileiro. Isso indica que as alterações realizadas no formato do relatório do auditor não foram capazes de expurgar as diferenças de expectativa em auditoria, sejam elas oriundas da deficiência nos relatórios dos auditores ou devido ao nível de conhecimento/educação dos usuários.

Sob uma outra ótica, a manutenção do descompasso entre as expectativas dos auditores e a sociedade pode estar atrelada à falta de conhecimento dos auditores em relação aos seus deveres, falta de capacidade técnica ou falta de interesse por parte dos auditores por considerar determinadas obrigações não rentáveis (Lee et al., 2009). Ao se reduzir as atividades desenvolvidas, os auditores incorrem em menor tempo de trabalho, o que, consequentemente, reduz os custos de auditoria e pode incrementar os lucros da firma.

Após verificada empiricamente a existência das diferenças de expectativa, investigou-se a possível existência de diferença de média entre o Índice de Expectativa do grupo que lê o relatório dos auditores comparado ao que não lê, dado que o grau de expectativa pode estar relacionado ao nível de utilização dos relatórios e a familiaridade com os mesmos. Os resultados são demonstrados na Tabela 4.

Apesar de apresentar diferenças entre a soma dos postos de ambos os grupos, os resultados apontaram a inexistência de diferença significativa entre o Índice de Expectativa daqueles que leem o relatório comparado aos que não leem. Expectativas exacerbadas e irreais sobre os deveres dos auditores poderiam ser esperadas por quem não lê o respectivo relatório. Contudo, constatou-se o contrário: aqueles que não leem o relatório apresentaram expectativas inferiores, o que pode ser resultado do fato de que quem não utiliza o relatório, não cria grandes exigências acerca dos deveres dos auditores. Cabe ressaltar, entretanto, que a diferença resultante do teste não foi estatisticamente significativa. Resultados similares foram detectados ao se realizar a análise daqueles que indicaram já ter lido o novo relatório dos auditores, dado 
pela adoção da revisão das normas internacionais de auditoria ISAs 700, 701, 705 e 706, em comparação aos que não leram.

Tabela 4:

Diferença de média por tipo de uso do relatório

\begin{tabular}{|c|c|c|c|c|c|c|c|c|}
\hline \multirow[b]{2}{*}{ Grupo } & \multicolumn{4}{|c|}{ Auditores e Não-Auditores } & \multicolumn{4}{|c|}{ Não-Auditores } \\
\hline & Obs & Soma dos Postos & Valor Esperado & Valor-P & Obs & Soma dos Postos & Valor Esperado & Valor-P \\
\hline Leio & 109 & 7.442 & 7.068 & \multirow{2}{*}{0.2964} & 69 & 5.173 & 5.037 & \multirow{2}{*}{0.5901} \\
\hline Não Leio & 76 & 9.763 & 10.137 & & 76 & 5.412 & 5.548 & \\
\hline Total & 185 & 17.205 & 17.205 & & 145 & 10.585 & 10.585 & \\
\hline
\end{tabular}

Fonte: Dados da pesquisa

Em seguida, analisou-se a possível existência de diferença significativa do Índice de Expectativa pelo critério de gênero, conforme demonstrado na Tabela 5.

Tabela 5:

Diferença de média por gênero

\begin{tabular}{lcccc}
\multicolumn{1}{c}{ Grupo } & Observações & Soma dos Postos & Valor Esperado & Valor-P \\
\hline Feminino & 82 & $8.217,5$ & 7.626 & 0,1020 \\
Masculino & 103 & $8.987,5$ & 9.579 & \\
\hline Total & $\mathbf{1 8 5}$ & $\mathbf{1 7 . 2 0 5}$ & $\mathbf{1 7 . 2 0 5}$ & \\
\hline
\end{tabular}

Fonte: Dados da pesquisa

Observa-se, na Tabela 5, que não existe diferença significativa entre o Índice de Expectativa quando analisado entre os gêneros feminino e masculino. Apesar disso, verifica-se que a soma dos postos do gênero feminino é superior ao esperado, enquanto, no caso masculino, observa-se o inverso. A expectativa superior do gênero feminino pode ser explicada pelo fato de que as mulheres podem ter sido submetidas a práticas educativas mais intensas do que os homens, por parte de seus pais, o que as levou a apresentarem escores mais elevados em algumas escalas, especialmente as relacionadas a exigência (Costa, Teixeira \& Gomes, 2000). Ainda assim, a diferença não foi suficientemente expressiva.

Trabalhos como o de Lundgren e OldenBorg (2016) - na Suiça - e de Maseko (2016) - na África do Sul - detectaram um impacto da adoção das normas internacionais de auditoria revisadas nas diferenças de expectativas em auditoria. Contudo, diante da indisponibilidade de dados históricos, o estudo não foi capaz de avaliar se a revisão das normas internacionais de auditoria foi capaz de reduziu significativamente as diferenças de expectativas em auditoria. Trabalhos nesse sentido foram conduzidos por intermédio de uma abordagem experimental, metodologia que não foi a adotada na presente pesquisa.

A persistência de diferenças de expectativa, no entanto, vai ao encontro de trabalhos posteriores à norma, que sugeriram que as diferenças de expectativas na auditoria persistem, mesmo com a transação de normas (Best et al., 2001; Gold et al., 2012; Sule et al., 2019; Dung \& Dang, 2019; Akther et al., 2019). Justificativa para referido fenômeno pode ser encontrada em estudos realizados por Almeida e Colomina (2008), Salehi et al. (2009), Gold et al. (2012), Kamau (2013), que têm apontado que o componente de maior representatividade na composição das DEA é dado pelas expectativas pouco razoáveis dos usuários. Wanderley (2017) também identificou a mesma tendência no Brasil, diante da detecção de expectativas não razoáveis dos usuários das demonstrações contábeis e de uma demanda por um maior quantitativo de informações em relação ao trabalho de auditoria.

Portanto, diante das recentes e significativas alterações da norma, percebe-se a necessidade de implantação de estratégias educativas pelos órgãos reguladores, com o intuito de explicitar ao usuário da auditoria quais são os deveres dos auditores e ampliar a compreensão dos limites e pontencidades do relatório apresentado pela auditoria. A capacidade do ensino de auditoria em contribuir positivamente na redução das DEA foi atestada por Nuernberg (2020), que, em um estudo realizado com 162 alunos, obteve indícios da redução de expectativas em questões referentes, principalmente, ao processo de auditoria, responsabilidades, bem como proibições e regulamentos das empresas de auditoria.

\section{Considerações Finais}

O estudo examinou a atual situação das diferenças de expectativas em relação aos deveres da auditoria externa após a adoção da revisão das normas internacionais de auditoria (ISAs 700, 701, 705), que culminou em atualizações no Relatório dos Auditores, no Brasil, no ano de 2017. Estudos relacionados às expectativas dos auditores e sociedade oferecem evidências empíricas, inputs e possibilidades de melhorias para as normas de auditoria vigentes, seja como resposta a crises e falhas referentes à confiabilidade da informação contábil, ou como estratégia de prevenção a estes fatos. 
O nível de exigência dos usuários da contabilidade em relação aos deveres dos auditores, captado pela proxy Índice de Expectativa (IE), foi calculado com base na metodologia desenvolvida por Porter (1993), utilizada em diferentes estudos (Chong, Pflugrath \& 2008; Albuquerque, 2009; Gold et al., 2012), estruturada pela soma de três componentes, sendo: responsabilidades que a sociedade espera dos auditores e o que é razoável esperar pelos seus trabalhos; responsabilidades razoáveis que a sociedade espera dos auditores e as responsabilidades determinadas por normas e leis; desempenho que o auditor deveria atender, de acordo com as normas e leis, e o desempenho percebido pela sociedade.

Questionários, elaborados com base nos trabalhos de Litjens et al. (2015), disponibilizados em meio eletrônico e aplicados presencialmente em dois congressos da área contábil no Brasil, captaram 194 respostas, das quais 9 apresentaram dados faltantes, restando, portanto, uma amostra final não probabilística de 185 respondentes, composta por 145 não-auditores (demais usuários das demonstrações contábeis) e 40 auditores.

Nesse rumo, a aplicação do teste de Mann-Whitney levou à rejeição da hipótese nula de comportamento equivalente entre os grupos, onde, neste caso, foi constatado que o índice de expectativa dos não-auditores em relação aos deveres da auditoria externa foi superior ao índice de expectativa dos próprios auditores independentes. Os testes aplicados, por si só, trouxeram resultados que contribuem à literatura, ao destacar a perpetuação de evidências empíricas documentadas nas últimas décadas. Demonstrou-se que, após a alteração da norma, que trouxe melhorias significativas ao relatório, diferenças de expectativas ainda podem ser detectadas, evidência que demonstra que somente a alteração da norma não é capaz de alterar a cultura do país e dos auditores.

Os resultados reforçam a necessidade contínua de treinamentos e de iniciativas educacionais, para que, de fato, os profissionais compreendam as informações divulgadas no relatório. A evolução do relatório deve ser constante, e possíveis melhorias devem ser sempre analisadas, buscando atender a mudanças de contexto e respondendo às demandas observadas na prática contábil. A partir de um treinamento e da difusão de informações acerca da função da auditoria, a sociedade poderá reduzir o nível de exigências não factíveis, fruto de expectativas pouco razoáveis, resultado da ignorância no que diz respeito à natureza dos trabalhos da auditoria, para focar no que, de fato, tem poder informacional ao usuário.

Para os auditores, a persistência das DEA encontradas na presente pesquisa explicita a necessidade de constante discussão sobre seu papel e como atender os anseios da sociedade. Em termos acadêmicos, 0 presente trabalho permite uma base de comparação para pesquisas subsequentes, que avaliem períodos posteriores à consolidação da norma. Dessa maneira, espera-se que, em um ciclo de retroalimentação, trabalhos científicos ofereçam insights para melhora da prática de auditoria.

Observa-se a necessidade de que trabalhos subsequentes incluam aspectos adicionais ao revisitar tais relações, tais como o nível educacional do respondente, tempo de experiência, características demográficas e quaisquer variáveis que possam interferir no nível de exigência informacional dos usuários do relatório da auditoria, inclusive características psicológicas. Tal análise requer a aplicação de estatísticas inferenciais, que permitem a inclusão de variáveis controle, isto é, de uma análise de regressão. Assim, poderse-ia considerar outras variáveis intervenientes no processo, ampliaria a riqueza, alcance e abrangência metodológica do estudo, reforçando a validade dos resultados empíricos encontrados por meio do teste de média.

A auditoria externa constitui-se como um dos mecanismos capazes de alinhar interesses, trazendo transparência e confiança entre os participantes envolvidos. Portanto, a discussão de como a informação é transmitida e recepcionada é benéfica para sociedade, permitindo que o debate seja extrapolado para possíveis futuros mecanismos de governança corporativa e desenvolvimento do mercado de capitais.

A compreensão das potencialidades informacionais do modelo atualizado de relatório de auditoria, no que tange à redução da existência do AEG, poderá ser ampliada em anos subsequentes, com acréscimo da série temporal de dados, e em um período de maior familiaridade da sociedade quanto à sua forma de leitura e conteúdo. A identificação da persistência das expectativas explicita a necessidade de constantes medidas para reduzi-la.

Dentre as limitações deste trabalho, destaca-se o tamanho da amostra e sua composição, que foi dada, predominantemente, por respondentes formados em Ciências Contábeis, podendo trazer possível viés aos resultados, uma vez que respondentes com esta formação podem ter cursado disciplinas de auditoria contábil, obtendo, assim, maior familiaridade com os deveres dos auditores estabelecidos em normas e leis, o que pode refletir em expectativas inferiores ao que seria encontrado em amostra diversa.

Ademais, diante da indisponibilidade de dados históricos, não foi possível avaliar o comportamento temporal das expectativas de auditoria. Sugere-se, para estudos futuros, o uso de experimentos, no intuito de avaliar o índice de expectativa dos usuários frente a diferentes alternativas de relatórios. Para tanto, ressaltase a necessidade de um ambiente controlado, permitindo que se captem as possíveis influências causadas pelas novas informações inseridas. 


\section{Referências}

Abram, L. (2006). Desigualdades de gênero e raça no mercado de trabalho brasileiro. Ciência e cultura, 58(4), 40-41.

Akther, T., Fengju, X., \& Haque, M. Z. (2019). An Investigation of Audit Expectation Gap in Bangladesh. Journal of Business, 4(2), 01-11. DOI: https://doi.org/10.18533/job.v4i2.120

Albuquerque, K. S. L. D. S. (2009). Auditoria e sociedade: análise das diferenças de expectativas dos usuários da contabilidade em relação ao papel do auditor.

Almeida, B. J. M. D. (2004). Auditoria e sociedade: o diálogo necessário. Revista Contabilidade \& Finanças, 15(34), 80-96. DOI: https://doi.org/10.1590/S1519-70772004000100006

Almeida, B. J. M. D., \& Colomina, C. I. M. (2008). Evidencia de las diferencias de expectativas en auditoria en mercados bursátiles de reducida dimensión: el caso Portugués. Revista Contabilidade \& Finanças, 19, 102-111. DOI: https://doi.org/10.1590/S1519-70772008000200009

Asare, S. K., \& Wright, A. M. (2012). Investors', auditors', and lenders' understanding of the message conveyed by the standard audit report on the financial statements. Accounting Horizons, 26(2), 193-217.DOI: https://doi.org/10.2308/acch-50138

Bédard, J., Gonthier-Besacier, N., \& Schatt, A. (2015). Analysis of the Consequences of the Disclosure of Key Audit Matters. Working Paper, Universite Laval.

Best, P. J., Buckby, S., \& Tan, C. (2001). Evidence of the audit expectation gap in Singapore. Managerial Auditing Journal. 6 (3), 134-144. DOI: https://doi.org/10.1108/02686900110385579

Boolaky, P. K., \& Quick, R. (2016). Bank directors' perceptions of expanded auditor's reports. International Journal of Auditing, 20(2), 158-174.DOI: https://doi.org/10.1111/ijau.12063

Boyd, D. T., Boyd, S. C., \& Boyd, W. L. (2001). The audit report: A "misunderstanding gap" between users and preparers. National Public Accountant, 45(10), 56-60.

Breesch, D., Hardies, K., \& De Muylder, J. (2012). The Added Value of Auditing in Belgium: Does Audit Remain If No Longer Mandatory?. Available at SSRN 2190396. DOI: http://dx.doi.org/10.2139/ssrn.2190396

Castro, P. R., Amaral, J. V., \& Guerreiro, R. (2019). Aderência ao programa de integridade da lei anticorrupção brasileira e implantação de controles internos. Revista Contabilidade \& Finanças, 30(80), 186201. https://doi.org/10.1590/1808-057×201806780

Chong, K. M., \& Pflugrath, G. (2008). Do different audit report formats affect shareholders' and auditors' perceptions?. International Journal of Auditing, 12(3), 221-241. DOI: https://doi.org/10.1111/j.10991123.2008.00381.x

Conselho Federal de Contabilidade - CFC (2016) NBC TA - de Auditoria Independente de Informação Contábil Histórica.

Coram, P. J., \& Wang, L. (2020). The effect of disclosing key audit matters and accounting standard precision on the audit expectation gap. International Journal of Auditing, 1-13. DOI: https://doi.org/10.1111/ijau.12203

Cordos, G. S., \& Fülöp, M. T. (2015). Understanding audit reporting changes: introduction of Key Audit Matters. Accounting \& Management Information Systems/Contabilitate si Informatica de Gestiune, 14(1).

Costa, F. T. D., Teixeira, M. A., \& Gomes, W. B. (2000). Responsividade e exigência: duas escalas para avaliar estilos parentais. Psicologia: reflexão e crítica, 13(3), 465-473.DOI: https://doi.org/10.1590/S0102$\underline{79722000000300014}$

Cruz, N. G., Lima, G. H., Durso, S., \& Cunha, J. V. A. (2018). Desigualdade de gênero em empresas de auditoria externa. Journal of Accounting, Management and Governance, 21(1), 142-159.

https://doi.org/10.21714/1984-3925 2018v21n1a8 
DeFond, M., \& Zhang, J. (2014). A review of archival auditing research. Journal of accounting and economics, 58(2-3), 275-326. DOI: https://doi.org/10.1016/j.jacceco.2014.09.002

Dung, N. N. K., \& Dang, A. T. (2019). The Study of Audit Expectation Gap: The Auditor's Responsibilities in a Financial Statement Audit in Vietnam. Asian Economic and Financial Review, 9(11), 1227. DOI: https://doi.org/10.18488/journal.aefr.2019.911.1227.1254

Dutra, M. H. (2011). Modelo de referência para o relatório final da auditoria independente baseado na abordagem de expectations gap.

Fonseca, V. H., Nunes, D. M. S., \& Santana, C. M. (2016). AMOSTRAGEM: Conhecimento e uso em empresas de auditoria. RAGC, 4(16).

Füredi-Fülöp, J. (2015). An empirical study of audit expectation gap in Hungary. Theory Methodology Practice: Club of Economics In Miskolc, 11(1), 37-46. DOI: https://doi.org/10.18096/TMP.2015.01.04

Geiger, M. A. (1994). Investor views of audit assurance: Recent evidence of the expectation gap. Journal of accountancy, 60.

Gold, A., Gronewold, U., \& Pott, C. (2012). The ISA 700 auditor's report and the audit expectation gap-Do explanations matter?. International Journal of Auditing, 16(3), 286-307.DOI: https://doi.org/10.1111/j.1099$\underline{1123.2012 .00452 . x}$

Gray, G. L., Turner, J. L., Coram, P. J., \& Mock, T. J. (2011). Perceptions and misperceptions regarding the unqualified auditor's report by financial statement preparers, users, and auditors. Accounting Horizons, 25(4), 659-684. DOI: https://doi.org/10.2308/acch-50060

Hassink, H. F., Bollen, L. H., Meuwissen, R. H., \& de Vries, M. J. (2009). Corporate fraud and the audit expectations gap: A study among business managers. Journal of international accounting, auditing and taxation, 18(2), 85-100.DOI: https://doi.org/10.1016/j.intaccaudtax.2009.05.003

IAASB - International Auditing and Assurance Standards Board (2011). Enhancing the Value of Auditor Reporting: Exploring Options for Change.

IAASB - International Auditing and Assurance Standards Board. (2013). Reporting on audited financial statements: Proposed new and revised International Standards on Auditing (ISAs).

Iwanowicz, T., \& Iwanowicz, B. (2019). ISA 701 and Materiality Disclosure as Methods to Minimize the Audit Expectation Gap. Journal of Risk and Financial Management, 12(4), 161. DOI:

https://doi.org/10.3390/irfm12040161

Kamau, C. G. (2013). Determinants of audit expectation gap: Evidence from limited companies in Kenya. International Journal of Science and Research (IJSR), 2(1), 480-491.

Lee, T. H., Ali, A. M., Gloeck, J. D., Yap, C. S., Ng, Y. L., \& Boonyanet, W. (2010). The audit expectation gap in Thailand. Southern African Journal of Accountability and auditing research, 10(1), 1-17.

Litjens, R., van Buuren, J., \& Vergoossen, R. (2015). Addressing Information Needs to Reduce the Audit Expectation Gap: Evidence from D utch Bankers, Audited Companies and Auditors. International Journal of Auditing, 19(3), 267-281. DOI: https://doi.org/10.1111/ijau.12042

Lundgren, L., \& Oldenborg, M. (2016). The public interest paradox of the Swedish auditing profession: A quantitative study of potential effects of the Swedish implementation of ISA 700 (revised) and ISA. Umeå School of Business and Economics.

Mafra, A. S., Cavalcanti, M. A. N., \& Penha, R. S. (2017). Fatores Condicionantes da Opinião Modificada nos Relatórios de Auditoria de Empresas Listadas na BM\&FBovespa. RAGC, 5(20).

Manoel, J., \& Quel, L. F. (2017). Innovation in the international standards for the new independent audit report. Journal on Innovation and Sustainability RISUS, 8(1), 130-143.DOI: http://dx.doi.org/10.24212/2179$\underline{3565.2017 v 8 \mathrm{i} 1 \mathrm{p} 130-143}$ 
Manson, S., \& Zaman, M. (2001). Auditor communication in an evolving environment: going beyond SAS 600 auditors'reports on financial statements. The British Accounting Review, 33(2), 113-136. DOI: https://doi.org/10.1006/bare.2001.0157

Maseko, K. N. (2015). Will the expanded audit report model proposed by IAASB reduce the expectation gap in South Africa? Master of Commerce in Accountancy, University of the Witwatersrand.

Menezes, A. L., \& Costa, F. M. (2012). Expectation Gap em auditoria: impactos no Brasil após a adoção do Projeto Clarity. Anais do XXXVI Encontro da ANPAD. Rio de Janeiro.

Mock, T. J., Bédard, J., Coram, P. J., Davis, S. M., Espahbodi, R., \& Warne, R. C. (2013). The audit reporting model: Current research synthesis and implications. AUDITING: A Journal of

Practice, 32(Supplement 1), 323-351. DOI: https://doi.org/10.2308/ajpt-50294

Nuernberg, V. M. (2020). O impacto do ensino da auditoria no Audit expectation GAP-estudo das Instituições de Ensino Superior de Pato Branco. Master's thesis, Instituto Politécnico do Cávado e Ave.

Nwaobia, A. N., Luke, O., \& Theophilus, A. A. (2016). The new auditors' reporting standards and the audit expectation gap. International Journal of Advanced Academic Research, 2(11), 118-133.

Oliveira, P. A. D. (2015). A mudança no relatório do auditor independente em resposta as expectativas de mercado: um auxílio na delimitação de sua responsabilidade?(Dissertação de Mestrado). Pontifícia Universidade Católica de São Paulo. São Paulo. Recuperado de https://tede2.pucsp.br/handle/handle/1618

Porter, B. (1993). An empirical study of the audit expectation-performance gap. Accounting and business research, 24(93), 49-68. DOI: https://doi.org/10.1080/00014788.1993.9729463

Porter, B., \& Gowthorpe, C. (2004). Audit expectation-performance gap in the United Kingdom in 1999 and comparison with the gap in New Zealand in 1989 and in 1999. Edinburgh, Scotland: Institute of Chartered Accountants of Scotland.

Salehi, M. (2011). Audit expectation gap: Concept, nature and trace. African Journal of Business Management, 5(21), 8376-8392. https://doi.org/10.5897/AJBM11.963

Salehi, M., Mansoury, A., \& Azary, Z. (2009). Audit independence and expectation gap: Empirical evidences from Iran. International Journal of Economics and Finance, 1(1), 165-174.

https://doi.org/10.5539/ijef.v1n1p165

Santos, E. S., \& Caldas, O. V. (2019, July). Diferença de expectativa na auditoria governamental. Anais do III CIDESP-Congresso Internacional de Desempenho do Setor Público.

Siegel, S., \& Castellan Jr, N. J. (2006). Estatística não-paramétrica para ciências do comportamento. Artmed Editora 2 ed.

Sterzeck, G. (2017). Audit expectation gap nos litígios das firmas de auditoria (Doctoral dissertation, Universidade de São Paulo). DOI: https://doi.org/10.11606/T.12.2017.tde-14062017-093241

Sule, S., Yusof, N. Z. M., \& Bahador, K. M. K. (2019). Users' perceptions on auditors' responsibilities for fraud prevention, detection and audit expectation GAP in Nigeria. Asian Journal of Economics, Business and Accounting, 1-10.DOI: https://doi.org/10.9734/AJEBA/2019/46832

Turner, J. L., Mock, T. J., Coram, P. J., \& Gray, G. L. (2010). Improving transparency and relevance of auditor communications with financial statement users. Current issues in auditing, 4(1), A1-A8. DOI: https://doi.org/10.2308/ciia.2010.4.1.A1

Veiga, J. G. T., Ribeiro, D. A. H., \& Inácio, H. C. (2017). O relatório de auditoria e as diferenças de expectativas em auditoria: Recentes alterações. Contabilidade e Gestão, 21, 155-177

Wanderley, L. E. T. L. (2017). As Diferenças de expectativas em auditoria no ambiente brasileiro. Doctoral dissertation, Fundação Getulio vargas, EBAPE. 


\section{NOTAS}

\section{AGRADECIMENTOS}

Os autores agradecem ao Centro de Pós-graduação e Pesquisas em Controladoria e Contabilidade do Departamento de Ciências Contábeis da UFMG

\section{CONTRIBUIÇÃO DE AUTORIA}

Concepção e elaboração do manuscrito: F.R. Pereira

Coleta de dados: F.R. Pereira

Análise de dados: F.R. Pereira

Discussão dos resultados: F.R. Pereira; R.T. Takamatusu, L. E. Taboada

Revisão e aprovação: R.T. Takamatusu, L. E. Taboada

\section{CONJUNTO DE DADOS DE PESQUISA}

O conjunto de dados que dá suporte aos resultados deste estudo não está disponível publicamente.

\section{FINANCIAMENTO}

Não se aplica.

\section{CONSENTIMENTO DE USO DE IMAGEM}

Não se aplica.

\section{APROVAÇÃO DE COMITÊ DE ÉTICA EM PESQUISA \\ Não se aplica.}

\section{CONFLITO DE INTERESSES}

Não se aplica.

\section{LICENÇA DE USO}

Os Direitos Autorais para artigos publicados neste periódico são do autor, com direitos de primeira publicação para a Revista. Em virtude de aparecerem nesta Revista de acesso público, os artigos são de uso gratuito, com atribuições próprias, em aplicações educacionais, de exercício profissional e para gestão pública. A Revista adotou a licença Creative Commons Atribuição 4.0 Internacional - CC BY NC ND. Esta licença permite acessar, baixar (download), copiar, imprimir, compartilhar, reutilizar e distribuir os artigos desde que com a citação da fonte, atribuindo os devidos créditos de autoria. Nesses casos, nenhuma permissão é necessária por parte dos autores ou dos editores. Autores têm autorização para assumir contratos adicionais separadamente, para distribuição não-exclusiva da versão do trabalho publicada nesta revista (ex.: publicar em repositório institucional ou um capítulo de livro).

\section{PUBLISHER}

Universidade Federal de Santa Catarina. Curso de Ciências Contábeis e Programa de Pós-graduação em Contabilidade. Publicação no Portal de Periódicos UFSC. As ideias expressadas neste artigo são de responsabilidade de seus autores, não representando, necessariamente, a opinião dos editores ou da universidade.

\section{EDITORES}

Carlos Eduardo Facin Lavarda e Suliani Rover

\section{HISTÓRICO}

Recebido em: 10/06/2020 - Revisado por pares em: 09/02/2021 - Reformulado em: 17/05/2021 Recomendado para publicação em: 18/05/2021 - Publicado em: 30/06/2021

\footnotetext{
*Preprint do artigo apresentado no XX USP International Conference in Accounting, 2020
} 